\title{
POSITIVITY OF DUALITY MAPPINGS
}

\author{
BY EDGAR ASPLUND
}

Communicated by F. Browder, September 21, 1966

The concept of a duality mapping was in troduced by Beurling and Livingston in [1]. A slight generalization of their definition follows.

Definition. A mapping $T$ from a normed linear space $E$ to its conjugate $E^{*}$ is called a duality mapping if the following two conditions are satisfied.

(1) The direction of $T(x)$ is conjugate to that of $x$ for all $x$ in $E$, i.e.

$$
\langle T(x), x\rangle=\|T(x)\|\|x\| .
$$

(2) There exists an increasing function $\phi$ from $R^{+}$to $R^{+}$such that

$$
\phi(\|x\|-0) \leqq\|T(x)\| \leqq \phi(\|x\|+0),
$$

defining $\phi(-0)=0$.

The main theorem of [1] is given below as Theorem 3, with a short, nonconstructive proof. In [2], [3], F. E. Browder has derived the Beurling-Livingstone theorem as a special case of a theorem on monotone operators, i.e. mappings $T$ from $E$ in to $E^{*}$ that satisfy the relation

(n) $\sum_{k \in Z_{n}}\left\langle T\left(x_{k}\right), x_{k}-x_{k-1}\right\rangle \geqq 0$ for all $\left(x_{1}, \cdots, x_{n}\right) \in E^{n}$ for $n=2$.

Duality mappings are monotone, in fact, they satisfy relation $(\mathrm{n})$ for all natural numbers $n$. We will call such mappings positive symmetric. $\mathrm{R}$. T. Rockafellar has proved in [4] that a mapping $T$ is positive symmetric if and only if it is the subgradient of some convex function $G$ defined on $E$, i.e. if

$$
G(y) \geqq G(x)+\langle T(x), y-x\rangle \text { for all } x, y \in E .
$$

The primitive $\Phi$ of $\phi$, defined by

$$
\Phi(t)=\int_{u=0}^{t} \phi(u) d u \text { for all } t \text { in } \overline{R^{+}}=R^{+} \cup\{0\}
$$

is convex, positive, and increasing. The following theorem thus shows that duality mappings are positive symmetric.

TheOREM 1. A mapping $T: E \rightarrow E^{*}$ is a duality mapping if and only if for all $x$ in $E, T(x)$ is a subgradient at $x$ of the convex function $\Phi(\|x\|)$, i.e.

$$
\Phi(\|y\|) \geqq \Phi(\|x\|)+\langle T(x), y-x\rangle \text { for all } y \text { in } E .
$$


Proof. Suppose that $T$ satisfies (3). Let $y$ vary subject to the restriction $\|y\|=\|x\|$. This yields

$$
\langle T(x), x\rangle \geqq\|T(x)\|\|x\| .
$$

Hence $T$ satisfies (1). Use this in (3) with $y=t z, x=s z,\|z\|=1$, and with $t$ and $s$ in $R^{+}$.

$$
\Phi(t) \geqq \Phi(s)+\|T(s z)\|(t-s) .
$$

This proves (2) on the ray from 0 through $z$. Conversely, suppose that $T$ is a duality mapping. Then by (1),

$$
\Phi(\|x\|)+\langle T(x), y-x\rangle \leqq \Phi(\|x\|)+\|T(x)\|(\|y\|-\|x\|)
$$

whereas by (2),

$$
\Phi(\|x\|)+\|T(x)\|(\|y\|-\|x\|) \leqq \Phi(\|y\|)
$$

so that (3) holds, as claimed.

The next theorem, which is the main result of this note, is a partial converse of Theorem 1.

Theorem 2. If $\phi(+0)=0$, then a mapping $T: E \rightarrow E^{*}$ that satisfies (2) is a duality mapping if and only if it is positive symmetric.

Proof. We have already seen that the condition is necessary. Suppose, then, that $T$ is positive symmetric. Let $G$ be the real valued convex function on $E$ of which $T$ is pointwise the subgradient. We call $G$ the potential of $T$ and we may suppose that $G$ is nonnegative and that $G(0)=0$. In fact, if $z$ is an arbitrary element of norm one in $E$, we may define $G$ by

$$
G(t z)=\int_{u=0}^{t}\langle T(u z), z\rangle d u \text { for all } t \geqq 0 .
$$

Because of (2), we know that the function $f$ defined on $R^{+}$by

$$
f(t)=\Phi(t)-G(t z)
$$

is nonnegative and nondecreasing. Now let $y$ be another element of norm one in $E$ and apply the subgradient relation for $G$ at $t z$ :

$$
\Phi(t) \geqq G(t y) \geqq G(t z)+\langle T(t z), t y-t z\rangle .
$$

We take the supremum of the right-hand side, as $y$ varies, and we obtain

$$
f(t) \geqq t\|T(t z)\|-t\langle T(t z), z\rangle
$$


Integrating this inequality over $t$ from 0 to $x$ and doing one partial integration on the right-hand side, we finally arrive at the inequality

$$
2 \int_{0}^{x} f(t) d t \geqq x f(x) \text { for all } x \text { in } R^{+} .
$$

For an arbitrary $\epsilon>0$, define $f_{\mathrm{e}}$ by $f_{\mathrm{e}}(x)=f(x)-\epsilon x$. Then

$$
2 \int_{0}^{x} f_{\mathrm{c}}(t) d t \geqq x f_{\mathrm{e}}(x) \text { for all } x \text { in } R^{+}
$$

holds also. However, the condition $\phi(+0)=0$ implies that $f_{\mathrm{e}}$ is strictly negative in some open interval with left end point zero. Because of (4), this interval must be $R^{+}$. But $f$ is nonnegative, so it must vanish identically, which proves that $G(y)=\Phi(\|y\|)$ for all elements $y$ in $E$, hence by Theorem 1 that $T$ is a duality mapping, as asserted.

Remark 1. Some condition like $\phi(+0)=0$ is needed. To see this, take for $\phi$ the function which is identically equal to one, take an element $y$ of unit norm in $E^{*}$ and define $T$ by

$$
\begin{aligned}
T(x) & =y \quad \text { if } \quad\langle y, x\rangle \geqq 0 \\
& =-y \quad \text { if } \quad\langle y, x\rangle<0 .
\end{aligned}
$$

Then $T$ is positive symmetric and satisfies (2), but it is not a duality mapping if $\operatorname{dim} E>1$.

REMARK 2. In finite dimensions, Theorem 1 can be interpreted as a theorem on the uniqueness of convex solutions of certain differential equations. As an example, let $E$ be euclidean and $\phi$ the identity function. Then the only convex solution $f$ of the differential equation

$$
\sum_{k=1}^{n}\left(\frac{\partial f}{\partial x_{k}}\right)^{2}=\sum_{k=1}^{n} x_{k}^{2}
$$

is (up to an additive constant)

$$
f=\frac{1}{2} \sum_{k=1}^{n} x_{k}^{2}
$$

There are, of course, several nonconvex solutions of (5).

REmark 3. Monotonicity of $T$ together with (2) does not imply that $T$ is a duality mapping. For, if $E$ is a Hilbert space and $\phi$ is the identity function, then all linear orthogonal mappings on $E$ with spectrum in the right half plane are monotone and satisfy (2), but the only duality mapping among these is the identity.

The last remark shows how much more general Browder's theorems 
on monotone operators in [2], [3] are than the theorem by Beurling and Livingston to which it specializes in the duality case:

Theorem 3 (BeurLing-Livingston). Suppose that $\lim _{t \rightarrow \infty} \phi(t)=\infty$ and that $F$ is a reflexive subspace of $E$. Let $F^{\perp}$ be the annihilator of $F$ in $E, v$ an element of $E$, and $w$ an element of $E^{*}$. Then there exists a duality mapping $T$ such that $T(x+v)$ is in $F^{\perp}+w$ for some $x$ in $F$.

Proof. Consider the convex function $f(x)=\Phi(\|x+v\|)-\langle w, x\rangle$ restricted to the reflexive space $F$. Since the function is weakly lower semicontinuous and tends to infinity as $\|x\| \rightarrow \infty$, uniformly in all directions, it assumes its minimum on $F$ at some point $x$ in $F$. By the Hahn-Banach theorem, $f$ considered as a function on all of $E$ has at $x$ some subgradient in $F^{\perp}$. By Theorem 1 , then, there exists a duality mapping satisfying the conditions of the theorem.

\section{REFERENCES}

1. A. Beurling and A. E. Livingston, $A$ theorem on duality mappings in Banach spaces, Ark. Mat. 4 (1962), 405-411.

2. F. E. Browder, On a theorem of Beurling and Livingston, Canad. J. Math. 17 (1965), 367-372.

3. F. E. Browder, Multivalued monotone nonlinear mappings and duality mappings in Banach space, Trans. Amer. Math. Soc. 118 (1965), 338-351.

4. R. T. Rockafellar, Characterization of the subdifferentials of convex functions Pacific J. Math. 17 (1966), 497-510.

University of Stockholm, SWEden 